\title{
A high-precision apparatus for the characterization of thermal interface materials
}

\author{
R. Kempers, ${ }^{1,2, a)}$ P. Kolodner, ${ }^{2}$ A. Lyons, ${ }^{2, b)}$ and A. J. Robinson ${ }^{1}$ \\ ${ }^{1}$ Department of Mechanical and Manufacturing Engineering, Trinity College Dublin, Dublin 2, Ireland \\ ${ }^{2}$ Alcatel-Lucent Bell Laboratories, Murray Hill, New Jersey 07974-0636, USA
}

(Received 7 January 2009; accepted 12 July 2009; published online 17 September 2009)

\begin{abstract}
An apparatus has been designed and constructed to characterize thermal interface materials with unprecedented precision and sensitivity. The design of the apparatus is based upon a popular implementation of ASTM D5470 where well-characterized meter bars are used to extrapolate surface temperatures and measure heat flux through the sample under test. Measurements of thermal resistance, effective thermal conductivity, and electrical resistance can be made simultaneously as functions of pressure or sample thickness. This apparatus is unique in that it takes advantage of small, well-calibrated thermistors for precise temperature measurements $( \pm 0.001 \mathrm{~K})$ and incorporates simultaneous measurement of electrical resistance of the sample. By employing precision thermometry, low heater powers and minimal temperature gradients are maintained through the meter bars, thereby reducing uncertainties due to heat leakage and changes in meter-bar thermal conductivity. Careful implementation of instrumentation to measure thickness and force also contributes to a low overall uncertainty. Finally, a robust error analysis provides uncertainties for all measured and calculated quantities. Baseline tests were performed to demonstrate the sensitivity and precision of the apparatus by measuring the contact resistance of the meter bars in contact with each other as representative low specific thermal resistance cases. A minimum specific thermal resistance of $4.68 \times 10^{-6} \mathrm{~m}^{2} \mathrm{~K} / \mathrm{W}$ was measured with an uncertainty of $2.7 \%$ using a heat transfer rate of $16.8 \mathrm{~W}$. Additionally, example measurements performed on a commercially available graphite thermal interface material demonstrate the relationship between thermal and electrical contact resistance. These measurements further demonstrate repeatability in measured effective thermal conductivity of approximately 1\%. () 2009 American Institute of Physics.
\end{abstract}

[doi:10.1063/1.3193715]

\section{INTRODUCTION AND BACKGROUND}

When heat is conducted between two solid surfaces in contact with one another, there exists a thermal contact resistance due to surface irregularities and asperities present at various length scales at the surfaces of the two materials. These surface imperfections limit the actual physical contact area at the microscale and form air gaps which often result in undesirable impedance to heat transfer between the two mating solid surfaces. One strategy to reduce thermal contact resistance is to employ a thermal interface material (TIM) to conform to the contacting surfaces and displace any microand macroscopic air voids, thereby providing a path of improved heat conduction which reduces the effective contact resistance.

In many high thermal energy dissipating systems, this TIM can account for up to $50 \%$ of the available thermal budget of the package. ${ }^{1}$ With the inevitable implementation of high-performance liquid cooling strategies, this percentage will become even greater. The improper characterization and/or implementation of TIMs can be very problematic

\footnotetext{
a) Author to whom correspondence should be addressed. Electronic mail: kempers@alcatel-lucent.com.

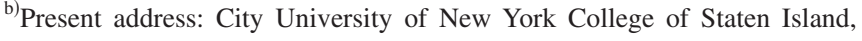
New York USA.
}

since, if the thermal management of an electronic device is inadequate, unacceptable temperature levels may be reached which can adversely affect device performance, reliability, and lifespan. ${ }^{1}$ These thermal issues have spawned a global effort towards the development of novel TIMs with complex formulation and very high performance. ${ }^{2-4}$

To work effectively in the emerging thermal environment, TIMs must conform to the mating surfaces under reasonable assembly pressures, have a thin bond line, and have low contact resistance with adequate bulk thermal conductivity. As TIM technology evolves, this combination of factors makes accurate quantification of the thermal characteristics of new generation materials difficult, as some of the most important quantities that need to be measured are dropping to levels below the uncertainty floor of conventional measurement methodologies. This poses very serious problems for the electronics packaging community since accurate knowledge of the thermal, mechanical, and electrical performance of TIMs, as well as the associated uncertainties in these values, is essential to the proper design of electronic systems and packages.

A number of physical properties must be known in order to fully characterize TIMs. They include, but are not limited to, thermal conductivity, thermal contact resistance, mechanical strength, compressibility and resilience, thermal sta- 
bility, coefficient of thermal expansion, dielectric strength, and breakdown voltage. ${ }^{5}$ Typically, for conventional TIMs, the contact resistance can be obtained experimentally by measuring several thicknesses of TIM, plotting the thermal resistance as a function of thickness, and extrapolating contact resistance as the $y$-intercept where the thickness is zero. ${ }^{6}$ However, for some next-generation TIMs such as metal microtextured (MMT) TIMs or carbon nanotubes, this method cannot be used due to the nonuniform behavior of the bulk TIM. Since it is experimentally difficult to distinguish between the bulk thermal resistance and contact thermal resistances of these next-generation TIMs, and since they are highly electrically conductive, it has been proposed that a measurement of electrical contact resistance be used in conjunction with a correlation with thermal contact resistance to infer the thermal contact resistance of these TIMs. ${ }^{7}$ With regard to thermal resistance and conductivity measurements, the state of the art can be broadly cast into two camps, transient techniques and static techniques, each with its respective niche. ${ }^{1}$

Dynamic- or transient-type TIM testers rely on the transient thermal response of a TIM to calculate their thermal resistance. Bosch and Lasance ${ }^{8}$ described a method of characterizing the thermal conductivity of TIMs based on earlier work performed by Lasance and Lacaze $^{9}$ using transient techniques. This setup consists of the TIM sample squeezed between an aluminum cooling/heating water jacket and an insulated copper block. The two metal blocks were instrumented with thermocouples. The water supply to the cooling/ heating baths was manually selectable between reservoirs at ambient temperature and at an elevated temperature. By introducing a step heat input through switching the water supply, the transient temperature response of the system could be measured. The effective thermal resistance of the sample was obtained by fitting the response data to predictions made with a numerical model of the system using the temperature data as boundary conditions. The authors quoted a reproducibility of $0.02 \mathrm{~K} / \mathrm{W}$, which corresponds to a specific thermal resistance of $2.51 \times 10^{-5} \mathrm{~m}^{2} \mathrm{~K} / \mathrm{W}$ for the sample area employed. Later work by Lasance et al. ${ }^{10}$ described this setup as having repeatability in the range from $1 \times 10^{-8}$ to $1 \times 10^{-7} \mathrm{~m}^{2} \mathrm{~K} / \mathrm{W}$ and a reproducibility of $2 \times 10^{-7} \mathrm{~m}^{2} \mathrm{~K} / \mathrm{W}$. However, they point out that these accuracies were determined through the procedure used to extract the interface resistance from the experimental results, not taking into account any actual experimental and measurement uncertainties.

Rencz and Székely ${ }^{11}$ and Rencz et al. ${ }^{12}$ further detailed the method in which the thermal resistance can be determined through a transient approach. They describe the use of the differential structure function which is calculated by direct transformations from a heating or cooling curve, measured as the thermal response of the system for a step function excitation. Local maxima in these functions yield information about the magnitude of the thermal resistance and capacitance of the different components in the thermal resistance path from the point of excitation and describe the heat flow path based on the geometry and material properties of the structure.
Smith et al. ${ }^{13}$ and Smith et al. ${ }^{1}$ discussed a number of difficulties with transient TIM testing. Among them is the inability of the power supply to provide ideally rectangular power steps, the slow change in heater resistance with temperature change, electrical noise, and heat spreading outside the one-dimensional path. Additionally, there has been little work performed to directly quantify the uncertainty in measurements obtained through these test methods. Still, at the expense of high temporal resolution sampling hardware and advanced data processing software, this technique offers the advantage of rapid testing in situ. ${ }^{1}$

Another popular transient technique for determining the thermal diffusivity of solid homogeneous materials is the laser flash method, which was originally proposed by Parker et al. ${ }^{14}$ Since then, improvements have been made to the technique and data reduction techniques. ${ }^{15-17}$ However, the density and heat capacity must be known, and indeed measured independently, in order to calculate the thermal conductivity of the TIM. ${ }^{16}$ These additional measurements and sources of error can lead to uncertainties in the thermal conductivity. ${ }^{5,18}$ A recent study employing the laser flash method to measure the performance of TIMs incurred uncertainties up to $25 \% .{ }^{17}$ Additionally, this method is unsuitable for measuring specimens of nonuniform thickness and has had limited success in characterizing the thermal resistance of a TIM in conjunction with contacting surfaces (or apparent thermal resistance), ${ }^{17}$ which is an important consideration for the characterization of TIMs.

Static testing is the second major approach to quantifying TIM thermal performance. Variants of the static test technique following ASTM D5470, "Standard test method for thermal transmission properties of thermally conductive electrical insulation materials," 19 concern measurement of the effective thermal conductivity or specific thermal resistance of thin conductive materials ranging from liquid compounds to hard solid materials. This technique has emerged as the de facto standard for characterizing TIMs by both researchers and manufacturers of TIMs. This standard describes a test procedure wherein heat is conducted between two parallel, isothermal surfaces separated by a test specimen of uniform thickness. The apparent thermal conductivity-the summation of bulk conductivity of the sample and the contact resistance-is calculated based on the temperature difference between the two surfaces and the imposed heat flux. While ASTM D5470 was not originally developed specifically for TIM characterization per se, recent revisions of this standard have addressed a number of concerns presented in previous studies. ${ }^{5,20}$ In particular, ASTM D5470-06 includes provisions for force and in situ thickness measurements during the application of load. ${ }^{19}$

While not the only possible implementation of ASTM D5470, a common experimental approach to obtaining the required data is represented schematically in Fig. 1. In this configuration, the sample is squeezed between two meter bars. Heat is supplied at one end and dissipated at the other. Temperature measurements made by sensors arrayed along the length of the meter bars are used to extrapolate the temperature at the contact surfaces. Additionally, with accurate knowledge of the thermal conductivity of the meter bars and 


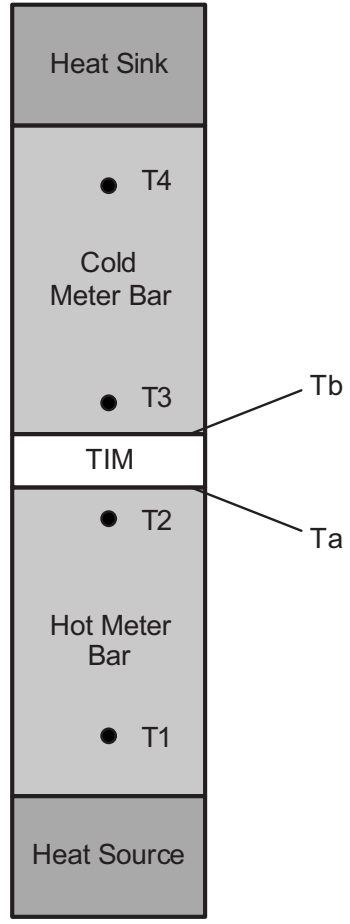

FIG. 1. Meter-bar implementation of ASTMD5470-06.

adequate insulation, the heat conducted through the sample can be calculated. Alternatively, the structure can be guard heated and the heat flux quantified by the electrical power into the primary heaters. The apparent specific thermal resistance (often referred to as thermal impedance) of the TIM is calculated as

$$
R A=\frac{A\left(T_{a}-T_{b}\right)}{Q},
$$

where $A$ is the cross-sectional area of the meter bars, $T_{a}$ and $T_{b}$ are the extrapolated contact surface temperatures, and $Q$ is the heat transfer rate. The effective thermal conductivity of the joint can then be calculated using

$$
k_{\text {eff }}=\frac{Q L}{A\left(T_{a}-T_{b}\right)}=\frac{L}{R},
$$

where $L$ is the thickness of the specimen bond line.

It is also important to point out that the schematic of the approach shown in Fig. 1 represents one engineering solution that can be used to impose the required test conditions and accomplish the necessary measurements. ${ }^{19}$ Although this design is not a unique implementation of the standard, many previous studies have used variations of this steady-state, heat-flux meter-bar approach. ${ }^{18,20-25}$

Gwinn et al. ${ }^{18}$ developed an apparatus for testing TIMs capable of measuring specific thermal resistances as low as $6.5 \times 10^{-6} \mathrm{~m}^{2} \mathrm{~K} / \mathrm{W}$ with a reported uncertainty of $10 \%$. The copper meter bars were $38.1 \mathrm{~mm}$ square in cross section and $45 \mathrm{~mm}$ long, instrumented with three 1.59-mm-diameter resistance temperature detectors (RTDs), with the closest RTD located $2 \mathrm{~mm}$ from the surface. Here, the RTDs were calibrated to $0.05 \mathrm{~K}$ with a thermal resolution of $0.026 \mathrm{~K}$. Heater powers up to $30 \mathrm{~W}$ were employed to achieve a measurable temperature difference across the interface. Heat losses were estimated as 30\%. A basic error analysis was presented based on these uncertainties to estimate the uncertainty in the thermal resistance. No provisions were included to measure sample thickness in situ.

Kearns ${ }^{20}$ adapted a commercially available conductivity tester to measure TIM thermal resistance with improved precision. This was due partly through the addition of precision calibrated RTDs and through modification of the apparatus to use meter bars for an improved measurement of surface temperature. This improved test facility measured specific thermal resistances as low as $3 \times 10^{-6} \mathrm{~m}^{2} \mathrm{~K} / \mathrm{W}$ with $10 \%$ uncertainty. Temperature resolution for this setup was $0.01 \mathrm{~K}$. Heater powers of $25-50 \mathrm{~W}$ were applied to achieve the necessary temperature difference.

Culham et al. $^{21}$ detailed the design and commissioning of a heat-flux meter-bar TIM tester and examined a number of issues pertaining to heat loss minimization, temperature measurement accuracy, and in situ thickness measurement. In this design, heat loss to the environment was minimized by enclosing the entire apparatus in a bell jar evacuated to a pressure of $1 \times 10^{-4}$ torr. Aluminum alloy 2024 meter bars instrumented with RTDs were used to both extrapolate the temperature at the TIM surfaces and quantify the heat flux. A laser/detector system was used to measure the distance between the meter bars for an accurate in situ measurement of the TIM thickness. Savija et al. ${ }^{22}$ used empirical data obtained using this apparatus in conjunction with an analytical model to predict the thermophysical properties of commercially available graphite sheets. This study reported relative uncertainty in measured thermal resistances of $2.2 \%$ for the thickest specimens to $13.6 \%$ for the thinnest specimens at the highest contact pressure. This apparatus was modified to measure the thermal conductivity and contact resistance of adhesives and is one of the few studies to clearly quantify measured uncertainties by plotting error bars on the thermal resistance data. ${ }^{6}$

Rao et $a l .{ }^{23}$ developed an apparatus to measure thermal contact resistance between two copper contacts at various atmospheric conditions and temperatures. A similar setup was employed by Misra and Nagaraju ${ }^{24}$ to measure both electrical and thermal contact resistance of brass-brass contacts in order to study the stability of electrical contacts due to thermal effects. Temperature measurements were performed using $1.1 \mathrm{~mm}$-diameter T-type thermocouples embedded into 1.2 -mm-diameter holes while a digital multimeter was used to perform electrical contact resistance measurements. This setup was used to validate theoretical models for thermal and electrical contact resistance with good agreement but cannot be used to characterize the performance of TIMs due primarily to the lack of instrumentation to measure bondline thickness.

Singhal et $a .^{25}$ also developed an apparatus to measure thermal contact resistance between metal-to-metal contacts. As with previous setups, the sensitivity and precision of this apparatus are limited by the elemental thermal uncertainty in the thermocouples being used $(0.2 \mathrm{~K})$.

As the next generations of TIMs offer improved thermal performance and thinner bond lines, there is a fundamental necessity for higher precision and more sensitive quantifica- 
tion of TIM performance than what is possible using the reviewed methods. The sensitivity, precision, and accuracy of thermal resistance measurements obtained in previous setups are largely dominated by the elemental thermal uncertainties of the sensors used. Additionally, for low thermal resistance measurements, large heater input powers were required to achieve a measurable temperature difference at the interface. This results in a large temperature gradient along the meter bars and accordingly large heat losses to the ambient which is another major source of uncertainty for these types of apparatus. ${ }^{26}$ Furthermore, a detailed and robust uncertainty analysis must also be employed for accurate quantification of the uncertainties associated with the measured quantities and how these uncertainties propagate to the calculated thermal resistance and effective thermal conductivity of the TIM. Previous studies only touch on this issue and present little or no uncertainty data in their results.

The present work addresses these issues and presents an experimental facility designed and built to measure TIM apparent thermal resistance and effective thermal conductivity with unprecedented precision and sensitivity. The underlying approach relies on the proven steady-state technique of using well-characterized meter bars to both extrapolate the temperature at the surfaces in contact with the TIM and measure the heat flow through the sample as illustrated in Fig. 1. It is rooted in setups used in previous studies, in particular, the apparatus used in Refs. 6, 21, and 22 which has proven accurate and reliable for many investigations and reflects improvements to the experimental apparatus originally presented by Kempers et al. ${ }^{27}$

This particular apparatus is unique primarily because it takes advantage of precision resistance thermometry to measure the axial temperature distribution along the meter bars, similar to the techniques employed by Kolodner $e t$ al. ${ }^{28}$ This achieves a level of thermal precision an order-of-magnitude higher than previous apparatuses, allows use of low input powers to minimize uncertainties due to heat leakage to the ambient, and eliminates any uncertainty arising from variations in meter-bar thermal conductivity due to a large imposed temperature gradient. Provisions were also incorporated to ensure the accuracy of force and thickness measurements. A precision machined sliding and alignment mechanism ensures meter-bar alignment throughout the range of motion. Furthermore, this TIM characterization apparatus is unique in employment of a simultaneous electrical contact resistance measurement to help characterize the electrical conductance and electrical contact resistance of a TIM during compressive loading for the purpose of gaining insight into the relationships between thermal and electrical contact resistance for next-generation MMT-TIMs. Finally, a particularly robust uncertainty analysis has been employed in order to accurately quantify the uncertainties in all measured and calculated quantities.

\section{EXPERIMENTAL APPARATUS}

\section{A. Apparatus design}

A detailed scale drawing of the apparatus is shown in Fig. 2, while a schematic illustrating the instrumentation is

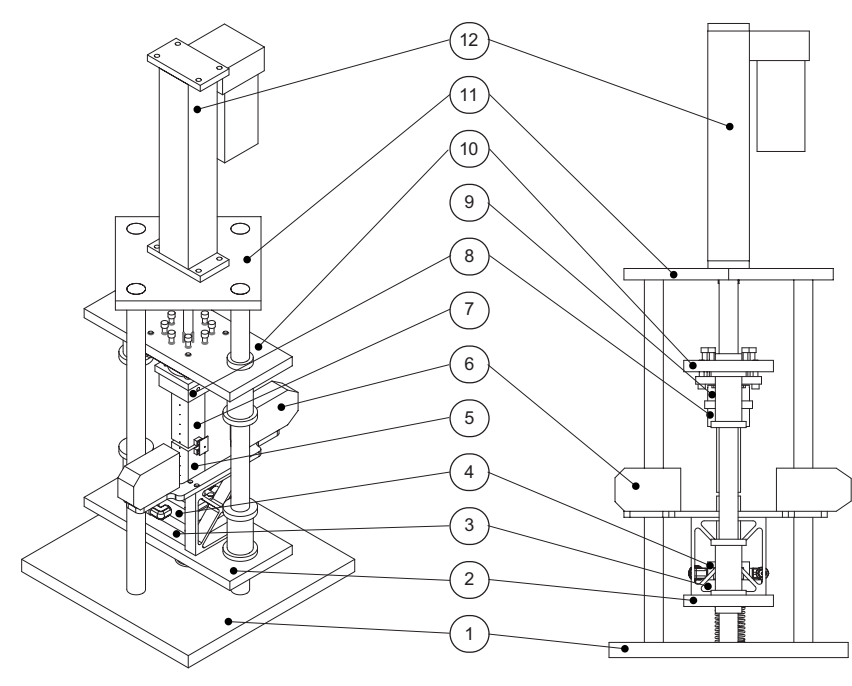

FIG. 2. Rendering of experimental facility developed in the present study.

shown in Fig. 3. The TIM sample under test is sandwiched between two $\mathrm{CuW}$ composite meter bars $120 \mathrm{~mm}$ long with contact areas of $40 \times 40 \mathrm{~mm}^{2}$. The lower meter bar (5) is bolted to the water jacket (4), which is cooled by a Julabo model F33 HE constant-temperature circulator. The circulator controller detects the temperature of a Pt100 sensor embedded in the water jacket, keeping its temperature stable to $\pm 0.01{ }^{\circ} \mathrm{C}$. The water jacket is situated atop a $25.4-\mathrm{mm}$-thick insulating block (3) and is bolted to the 19.05-mm-thick steel lower platen (2).

The upper meter bar (7) is bolted to the copper heater block (8) which contains two cartridge heaters. The heater block is mounted to a load cell (9) through a $12.7 \mathrm{~mm}$ steel plate. The load cell is then attached to the upper platen (10) using a similar $12.7 \mathrm{~mm}$ steel plate and a series of bolts that allow the upper meter bar to be fixed to the upper platen at a

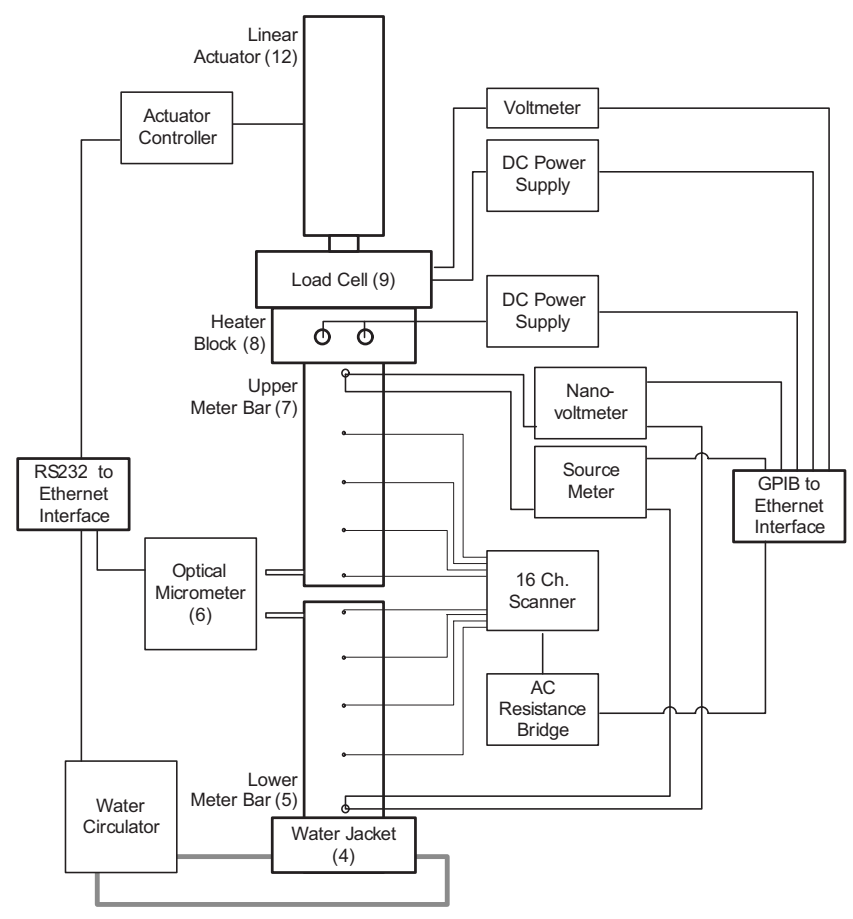

FIG. 3. Schematic of instrumentation and control of experimental facility. 
set position relative to the lower meter bar. The upper platen is also constructed from 19.1-mm-thick steel. The distance between the opposing faces of the meter bars is measured using an optical micrometer (6).

The upper and lower platens are fitted with ball bushings and slide on two precision-ground steel shafts, preventing any lateral motion. At the bottom, the shafts are attached to a 25.4-mm-thick steel base plate (1) of lateral dimensions $400 \times 400 \mathrm{~mm}^{2}$. At the top, the shafts are fastened together with a $19.05 \mathrm{~mm}$ steel plate (11). A linear actuator (12) is mounted to the top plate with the actuator arm attached to the upper platen. During testing, the meter bars are enclosed in $15 \mathrm{~mm}$ of Aspen Aerogel insulation (not shown) to further minimize heat leaks. The manufacturer's specification for the thermal conductivity of this insulation was $0.015 \mathrm{~W} / \mathrm{m} \mathrm{K}$.

A typical test procedure consisted in setting the circulator water temperature and input power to the heaters to desired values and incrementing the actuator until the desired displacement and thus starting pressure is realized. The system was allowed to reach steady state, whereupon the measurements were logged, the actuator was incremented a defined amount, and the process was repeated. All instrument control and data acquisition were performed using a personal computer with MATLAB software communicating to the instruments through serial and general purpose interface bus (GPIB) interfaces.

\section{B. Meter bars, thermal instrumentation, and calibration}

The meter bars in this apparatus were made from Elkonite copper-tungsten alloy 30W3 manufactured by CMW Inc. This alloy consists of $80 \% \mathrm{~W}$ and $20 \% \mathrm{Cu}$ by weight and consists of microsintered $\mathrm{W}$ foam filled with $\mathrm{Cu}$, resulting in a hard composite with thermophysical properties that are intermediate between those of the two pure metals and thus a better balance between hardness and thermal conductivity than either pure metal. This reduces the likelihood that the contact surfaces become scratched or worn over time and maintains a low thermal gradient in the meter bars. The hardness of this material was measured before and after final surface finishing and found to have a hardness of 102 Rockwell Hardness B. The manufacturer quotes an electrical resistivity of $42.1 \mathrm{n} \Omega \mathrm{m}$.

The surfaces in contact with the specimen were fly cut and ground in order to achieve a smooth, flat surface. The contact surfaces were characterized using a commercial white-light interferometer at several locations on each meter bar. Over a sample area of $3.7 \times 5 \mathrm{~mm}^{2}$, the lower meter bar had a first moment of roughness, or average absolute deviation from the mean of $113.7 \mathrm{~nm}$, a rms roughness of 152.5 $\mathrm{nm}$, and a peak-to-trough maximal roughness of $1.79 \mu \mathrm{m}$. The upper meter bar had a first moment of roughness of 78.2 $\mathrm{nm}$, a rms roughness of $105.5 \mathrm{~nm}$, and a maximal peak-totrough of $1.94 \mu \mathrm{m}$. To assess their overall flatness, the polished surfaces were contacted with an optical flat and illuminated with narrow-band green light. The resulting interference fringes were observed visually. The deviation from flatness did not exceed five fringes, which corresponds to
$2.5 \mu \mathrm{m}$. No falloff was observed at the edges of the polished faces.

Each meter bar was instrumented with four Betatherm thermistors measuring $2 \mathrm{~mm}$ long and $0.38 \mathrm{~mm}$ in diameter with a nominal resistance of $22 \mathrm{k} \Omega$ at $25{ }^{\circ} \mathrm{C}$. Thermistors were chosen for their high temperature sensitivity and small probe size, keeping spatial and thermal uncertainties to a minimum and thereby minimizing the uncertainty in successive calculated quantities of extrapolated temperature and heat flux. ${ }^{29}$ The thermistors were inserted into $0.4-\mathrm{mm}$ diameter holes of $3 \mathrm{~mm}$ depth at locations approximately 3 , 20, 40, and $60 \mathrm{~mm}$ from the contact surfaces. Following machining, the hole positions, hole diameters, and contact areas of both meter bars were measured optically using a precisely characterized opticomechanical measurement and alignment system with a precision of about $1 \mu \mathrm{m}$. Average hole diameter was $0.42 \mathrm{~mm}$, thus resulting in a uniform thermistor positioning uncertainty of $\pm 20 \mu \mathrm{m}$.

As in previous investigations, ${ }^{18,20}$ numerical simulations were employed to determine the influence of the intruding thermistors on the temperature profile through the meter bars and the associated heat fluxes and the temperature distributions at the contact surface. It was found that the thermistors closest to the contact surface yielded a negligible influence on temperature and heat-flux uniformity at the surface. Furthermore, these simulations demonstrated that heat leaks to the ambient had a negligible impact on the energy balance or the linearity of the axial temperature profiles in the meter bars.

The thermistors were held in place using a silicone adhesive. To minimize heat leaks through the thermistor leads, the lead wires were put in thermal contact with the meter bar at the same axial positions before making external connections. For strain relief, the lead wires from each thermistor were soldered to an adhesive-backed soldering strip attached to the side of each meter bar, where connections were made to the external instrumentation via fine $\mathrm{CuNb}$ wires to further minimize heat leaks to the environment. Thermistor resistances were measured using a LakeShore model 370 ac resistance bridge equipped with a 16-channel scanner, resulting in accurate and precise four-wire resistance measurements using ac excitation and low excitation current $(3.16 \mu \mathrm{A})$ to minimize thermistor self-heating. The temperature resolution of the present setup is approximately $1 \times 10^{-4}{ }^{\circ} \mathrm{C}$.

The effects of shot noise and Johnson-Nyquist noise were estimated based on the excitation current and nominal resistance values of the thermistors and found to be on the order of $0.02 \Omega$, significantly lower than the resolution of the current setup. The noise level was verified experimentally by measuring the resistances of several lowtemperature-coefficient resistors in a stable thermal environment continuously over $24 \mathrm{~h}$ and was indeed found be less than the measurement resolution.

Once installed, the thermistors on both meter bars were calibrated simultaneously against a Hart Scientific 5611T secondary reference probe whose absolute calibrated uncertainty was $\pm 0.002 \mathrm{~K}$. The reference probe was mounted inside a copper block bolted tightly to the upper and lower meter bars. The entire assembly was placed inside a 
170-mm-diameter, 300-mm-long copper cylinder closed at one end with a removable copper cap at the other end. Copper tubing was densely coiled around the outside of this calibration cylinder and soldered in place. Water, supplied by the constant-temperature circulator with a stability of $\pm 0.01 \mathrm{~K}$, was passed through the copper tubing to control the temperature of the calibration cylinder. The calibration cylinder was insulated with no less than $200 \mathrm{~mm}$ of foam insulation to maintain a stable thermal environment. The thermal mass of the calibration cylinder, combined with the imperfect thermal contact with the assembly inside, reduced these fluctuations to less than $0.001 \mathrm{~K}$ at the thermistor locations. The thermistors were calibrated against the secondary reference probe using six points over a range from 15 to $40{ }^{\circ} \mathrm{C}$ and curve fitted to a generalized Arrhenius form using a thirdorder polynomial. Between any temperature instabilities in the calibration environment and curve fitting errors, the relative temperature uncertainty measurement between the thermistors was reduced to $\pm 0.001 \mathrm{~K}$ with a confidence interval of two standard deviations.

Since the upper and lower meter bars were cast in separate batches and meter-bar thermal conductivity is vital to accurate quantification of heat flux through the sample, their thermal conductivities were carefully measured independently. This was achieved by electrically heating one end and fixing the temperature of the opposite end using the constanttemperature circulator and measuring the temperature gradient in the meter bars using the calibrated thermistors. Ample insulation and low heater powers ensured negligible heat loss to the ambient air. Estimates using numerical methods further supported this assumption and an uncertainty analysis, similar that presented in Sec. III, was used to quantify the uncertainty in the measured thermal conductivity. The upper and lower meter bars were found to have conductivities of $214 \pm 2$ and $216 \pm 2 \mathrm{~W} / \mathrm{m} \mathrm{K}$, respectively.

\section{Electrical contact resistance measurement}

Electrical connections for the four-wire resistance measurements were made by drilling a hole approximately $4 \mathrm{~mm}$ deep on one side of each meter bar approximately $5 \mathrm{~mm}$ from where they connected to a water jacket or heater block. The holes were tapped for M3 screws to allow leads to be clamped directly to the meter bars. Care was taken to ensure the meter bars were electrically isolated from the rest of the apparatus. A Keithley model 2400 sourcemeter was used to provide a constant current of up to $100 \mathrm{~mA}$ while a Keithley model 2182A nanovoltmeter was used to measure the corresponding voltage drop. A current-reversal method was used to minimize thermoelectric voltage offsets. ${ }^{30}$ The bulk electrical resistance of each meter bar was calculated to be $2.72 \mu \Omega$.

\section{In situ sample thickness measurement}

The sample thickness is measured optically using a Keyence model LS-7030 optical micrometer. Steel gauge pins, $2 \mathrm{~mm}$ in diameter and approximately $15 \mathrm{~mm}$ long, were mounted normal to the meter-bar surfaces near the contacting surfaces to act as optical trips for the micrometer. Prior to
TABLE I. Uncertainty in measured quantities.

\begin{tabular}{cc}
\hline \hline Measured quantity & Uncertainty \\
\hline Temperature & $\pm 0.001 \mathrm{~K}$ \\
Thermistor location & $\pm 20 \mu \mathrm{m}$ \\
Force & $\pm 0.2 \%$ of rated load \\
Meter-bar area & $\pm 9 \times 10^{-7} \mathrm{~m}^{2}( \pm 0.056 \%)$ \\
TIM thickness & $\pm 0.15 \mu \mathrm{m}$ \\
ECR current & $\pm 0.066 \%+20 \mu \mathrm{A}$ for $100 \mathrm{~mA}$ range \\
ECR voltage & $\pm .005 \%+40 \mathrm{nV}$ for $10 \mathrm{mV}$ range \\
\hline \hline
\end{tabular}

sample insertion, the micrometer was zeroed by bringing the meter bars into contact and measuring the distance between the optical trips. This setup allowed for the measurement of samples as thick as approximately $20 \mathrm{~mm}$ with an uncertainty of $\pm 0.15 \mu \mathrm{m}$. This method was favored over other methods such as linear variable differential transformer or traditional micrometers due to its noncontact nature and indifference to meter-bar thermal expansion or system deflection as loads are applied.

\section{E. Load application and measurement}

The upper platen assembly was displaced using an Industrial Devices model EC3 stepper-motor-controlled Acme screw linear actuator, capable of exerting $7200 \mathrm{kN}$ of force. The high-friction characteristics of the Acme screw prevent potential backdriving caused by the weight of the assembly. A controller capable of microstepping the stepper motor allowed for submicron changes in displacement to the platen.

The force applied to the sample is measured using an AST model KAF-S load cell with a rated load of $5 \mathrm{kN}$. The accuracy is limited by the load cell to $\pm 0.2 \%$ of rated load. Over the meter-bar area, this results in a maximum measurable pressure of $3.13 \mathrm{MPa}$. For lower-pressure testing, the load cell can be replaced with one with a lower rated load to achieve higher accuracies at low pressures.

Due to the employment of a stepper-motor-controlled linear actuator, the applied load is displacement controlled and is adequate for most testing procedures. When testing soft, compliant TIMs, the lower platen and meter bar are held rigidly in place, allowing deflection to occur in the TIM only. When testing rigid specimens or performing self-contact tests, the lower platen floats on an array of springs to allow the pressure to be varied linearly over a range of controlled displacements. Additionally, the apparatus is capable of pressure control and thickness control through a software control system relying on feedback from the load cell or optical micrometer.

\section{UNCERTAINTY ANALYSIS}

A rigorous uncertainty analysis was employed in order to quantify how the uncertainties of every measured quantity propagate to the overall uncertainty in the thermal resistance and effective thermal conductivity of the TIMs under test. Each measured quantity and its associated uncertainty are listed in Table I. 


\section{A. Calculated quantities}

The temperatures at the contact surfaces, $T_{a}$ and $T_{b}$, and the heat flux $Q$ for each meter bar were obtained by performing least-squares regression of the axial temperature distribution to a straight line and computing the resulting $y$-intercept and slope at the contact surfaces. As a result, the uncertainties in $T_{a}, T_{b}$, and $Q$ depend on both the thermal and spatial uncertainties of each thermistor.

There are a number of statistical approaches to calculating how the measured uncertainty propagates through a least-squares regression. Wald ${ }^{31}$ and Bartlett ${ }^{32}$ outlined methods for fitting a straight line when both variables are subject to error. These methods are mathematically involved and rely on the assumption that the uncertainties are uniform and normally distributed. Press et $a l .{ }^{33}$ attempted to describe an analytical method for calculating the uncertainties in the slope and $y$-intercept of a straight line model based on the assumption of a normal distribution and the standard deviations in both the $x$ and $y$ data. The resulting expressions are nonlinear and unwieldy and best suited to numerical solutions. ${ }^{33}$ Kedzierski and Worthington ${ }^{29}$ presented relatively straightforward expressions for estimates of the uncertainties in wall temperature and gradient as originally obtained by $\mathrm{Ku}^{34}$ These expressions demonstrate that the lowest uncertainties are obtained by using a meter bar of high thermal conductivity having a large number of well-spaced, small-diameter holes. They further demonstrate that the calculated surface temperature can have a greater precision than those of the individual temperature measurements. ${ }^{29}$

For the present study, the uncertainties in the $y$-intercepts and slope are computed numerically using a Monte Carlo simulation. Aside from the relative simplicity of this method, it is also advantageous in its ability to deal with nonuniform uncertainties among the temperature and position measurements as well as differing uncertainty distributions. Here, the temperature uncertainty is assumed to have a normal distribution where the uncertainty listed in Table I is equal to two standard deviations. Previous studies have suggested that the thermistor location uncertainty also has a normal distribution; however, this implies that it is statistically possible for the thermistor to be located outside the hole. ${ }^{29}$ Additionally, there is no physical justification for the location uncertainty to have a normal distribution. Other authors have argued that once the temperature sensors are fixed in place, this error is systematic and thus does not have as pronounced an effect. In the present study, we opted for the conservative yet realistic approach of modeling this uncertainty as a flat distribution bounded by difference in radii between the thermistors and the holes. The standard deviation for the slopes and the intercepts are calculated by performing 2000 randomized curve fits to the data constrained by the $x$ and $y$ uncertainty distribution at each point. The uncertainties in the slope and $y$-intercept are then taken as two standard deviations of this data set.

The heat transfer rate through each meter bar is then computed by

$$
Q_{\mathrm{mb}}=m_{\mathrm{mb}} k_{\mathrm{mb}} A,
$$

where $m_{\mathrm{mb}}$ is the temperature gradient (or slope) through each meter bar, $k_{\mathrm{mb}}$ is the thermal conductivity of each meter bar, and $A$ is the cross-sectional area of the meter bar. The apparent thermal resistance of the TIM is then calculated as

$$
R=\frac{\left(T_{a}-T_{b}\right)}{Q},
$$

where $Q$ is the mean heat transfer rate through the meter bars. The effective thermal conductivity of the joint can then be calculated using

$$
k_{\mathrm{eff}}=\frac{Q L}{A\left(T_{a}-T_{b}\right)}=\frac{L}{A R},
$$

where $L$ is the thickness of the specimen bond line.

The uncertainty in each quantity calculated in Eqs. (3)-(5) was obtained using the method of Kline and McClintock. ${ }^{35}$ Here, each measurement is denoted by $x_{i}$ and the uncertainty in the measurement $U_{i}$. The result of a calculation using these measurements is denoted $Z$, and the uncertainty in the calculated result is denoted by $U_{z}$. The uncertainty $U_{z}$ is then calculated as

$$
U_{z}=\sqrt{\left(\sum_{i=1}^{n}\left[\frac{\partial Z}{\partial x_{i}} U_{i}\right]^{2}\right)} .
$$

All calculated quantities and their associated uncertainties are computed in real time by the data acquisition software.

As an adjunct to this automated computation, it is useful to estimate and discuss the magnitudes of the different contributions to the total uncertainty $U_{R}$. In order of decreasing importance, they are as follows:

(1) At low values of the TIM thermal resistance $R$ and sufficiently high heater powers $Q$, the dominant uncertainty is incurred in extrapolating the temperature profile from the locations $d$ of the two thermistors closest to the contact surfaces to the surfaces themselves. The correction for each surface is $\Delta T=m_{\mathrm{mb}} d$, where $m_{\mathrm{mb}}$ is the measured temperature gradient and $d \approx 2.8 \mathrm{~mm}$ is the distance to the contact surface. The dominant uncertainty in $\Delta T$ is due to the imprecision $U_{d}= \pm 20 \mu \mathrm{m}$ in the thermistor positions, and this leads to a temperature uncertainty $U_{T}=\sqrt{2} m_{\mathrm{mb}} U_{d}$, where the factor $\sqrt{2}$ is contributed by the independent extrapolation of the temperature gradients in the two meter bars. Dividing this uncertainty by the temperature difference $T_{a}-T_{b}=Q R$ across the TIM gives the fractional uncertainty $f_{\text {ext }}$ in the TIM resistance:

$f_{\text {ext }}=\sqrt{2} U_{d} / k_{\mathrm{mb}} R A=1.3 \times 10^{-7} / R A$,

with $R A$ in units of $\mathrm{m}^{2} \mathrm{~K} / \mathrm{W}$. This result implies that this apparatus can measure specific thermal resistances as low as $2.6 \times 10^{-7} \mathrm{~m}^{2} \mathrm{~K} / \mathrm{W}$ with $50 \%$ fractional uncertainty. Improving this unprecedented sensitivity would require the use of smaller and more precisely located thermistors.

(2) If the TIM specific thermal resistance is increased above $1.4 \times 10^{-5} \mathrm{~m}^{2} \mathrm{~K} / \mathrm{W}$, the total uncertainty for suffi- 
ciently high heater powers $Q$ is dominated by the uncertainty in the independent measurement of the meter-bar conductivities $k_{\mathrm{mb}}$. This fractional uncertainty is $0.9 \%$. This component of uncertainty is systematic, not random. As such, it does not affect the sensitivity of the apparatus or comparisons between different measurements of thermal resistance.

(3) An upper bound on the fractional uncertainty contributed by the temperature dependence of the thermal conductivities of the meter bars can be estimated by multiplying the largest end-to-end temperature difference employed in our experiments by any estimate of temperature derivative of the conductivity. Since the meter bars are a $\mathrm{CuW}$ composite and not a true alloy, their conductivities are a concentration-weighted average of those of the pure metals. The larger temperature derivative is that of tungsten: $k^{-1} d k / d T=-7.5 \times 10^{-4} \mathrm{~K}^{-1}$. The largest temperature difference in these experiments is about $2 \mathrm{~K}$; thus, a very conservative upper bound for this component of fractional uncertainty is $0.15 \%$.

(4) The random noise $U_{T}$ in the thermistor signals is observed to be about $U_{T}= \pm 0.2 \mathrm{mK}$. These fluctuations dominate the total uncertainty at very low heater powers $Q$. The contribution of these fluctuations to the total fractional uncertainty in TIM resistance is

$f_{n}=k_{\mathrm{mb}} A U_{T} / 2 Q \Delta d=1.7 \times 10^{-3} / Q$,

with $Q$ in W. Here, $\Delta d=20 \mathrm{~mm}$ is the spacing between thermistors, and the factor 2 represents the averaging of four thermistor temperatures in computing the temperature gradient in the meter bars. This source of uncertainty becomes unimportant when $Q$ is increased above $0.19 \mathrm{~W}$, a very low-power level. The extremely low thermistor noise allows this apparatus to be operated with full precision at very low temperature gradients. This results in minimal perturbation of the apparatus by spatially nonuniform heat leaks to the environment.

(5) The uncertainty $U_{d}$ in the thermistor positions contributes a negligible fractional uncertainty $f_{d}=U_{d} / 2 \Delta d=5$ $\times 10^{-4}$ to the imprecision in the measured temperature gradients.

(6) The thermistor calibration uncertainty of $U_{\text {cal }}$ $= \pm 2 \mathrm{mK}$ makes an utterly negligible contribution to the total measurement uncertainty. Because the thermistors are all calibrated together, their readings are all affected identically by any distortions in the true temperature calibration of the reference thermistor. Thus, such distortions have a tiny effect on the measurement of temperature differences, which is the basis of our determination of the temperature gradients in the meter bars. A rough estimate of the fractional uncertainty contributed by this distortion is $f_{\text {cal }}=U_{\text {cal }} / \Delta T_{\text {cal }}$, where $\Delta T_{\text {cal }} \approx 20 \mathrm{~K}$ is the spacing between the calibration points supplied by the thermistor manufacturer. This estimate gives $f_{\text {cal }}=1 \times 10^{-4}$. Since the total temperature gradient in either meter bar is less than $10 \mathrm{~K}$ even at the highest power levels, the temperature uncertainty of \pm 1

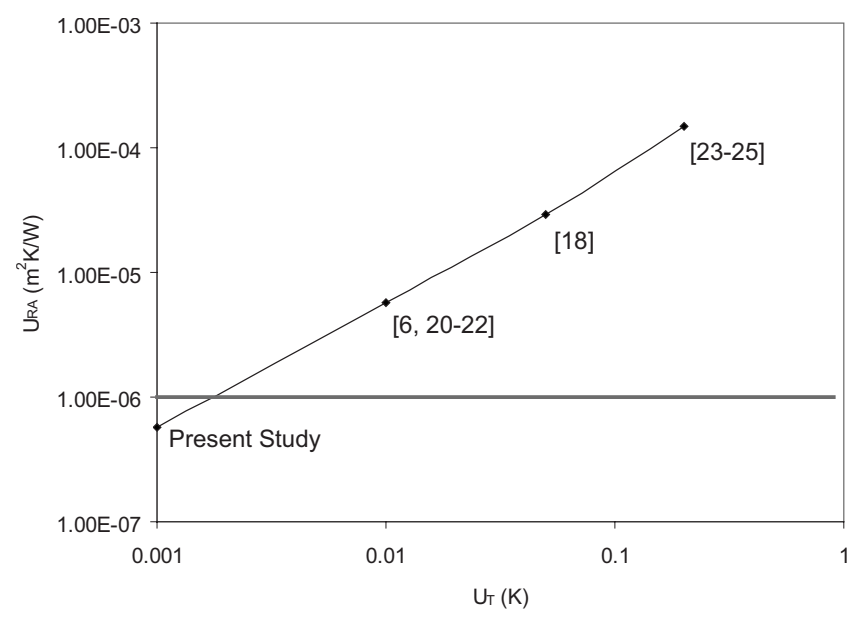

FIG. 4. Variation in specific thermal resistance uncertainty $U_{R A}$ with thermal sensor uncertainty $U_{T}$ for a common set of experimental parameters.

$\mathrm{mK}$ used in our Monte Carlo calculations is considered to be a conservative of the effect of thermistor calibration uncertainty.

\section{B. Comparison to previous apparatuses}

Ultimately, the precision and sensitivity of thermal resistance measurements obtained using the steady-state, heatflux meter-bar approach common to our apparatus and those described in the literature rely primarily on the magnitude of two elemental uncertainties, namely, the thermal uncertainties of the sensors employed and the uncertainties in their spatial locations. Direct comparisons between apparatuses are difficult due to the varying geometries, testing conditions, and information provided in each study. However, a simple comparison demonstrating the influence of only the thermal precision on the uncertainty in the measured specific thermal resistance, $U_{R A}$, can be made by applying Eq. (6) directly to Eq. (1). Here it is assumed that the temperature uncertainty at the contacting surfaces is equal to the elemental sensor uncertainty and that all other uncertainties are zero. This results in

$$
U_{R A}=\sqrt{\left(\frac{A}{Q}\right)^{2}\left(U_{\Delta T}\right)^{2}+\left(\frac{-A \Delta T}{Q^{2}}\right)^{2}\left(U_{Q}\right)^{2}},
$$

where

$$
U_{Q}=\sqrt{\left(\frac{k A}{\Delta x}\right)^{2}\left(U_{\Delta T}\right)^{2}}
$$

and

$$
U_{\Delta T}=\sqrt{U_{T a}^{2}+U_{T b}^{2}} .
$$

Thus, for given values of $k, A, \Delta x$, and $Q$, the variation in the uncertainty $U_{R A}$ in the specific thermal resistance [Eq. (9)] can be plotted as a function of sensor thermal uncertainty $U_{T}$. Figure 4 shows the magnitude of $U_{R A}$ evaluated for a common parameter set typical for our experiment $(k$ $=215 \mathrm{~W} / \mathrm{m} \mathrm{K}, A=1600 \mathrm{~mm}^{2}, \Delta x=20 \mathrm{~mm}$, and $Q=4 \mathrm{~W}$ ) using values of the thermal precision, $U_{T}$, corresponding to previous studies reported in the literature. The labels on the data points indicate the authors whose studies exhibited the 


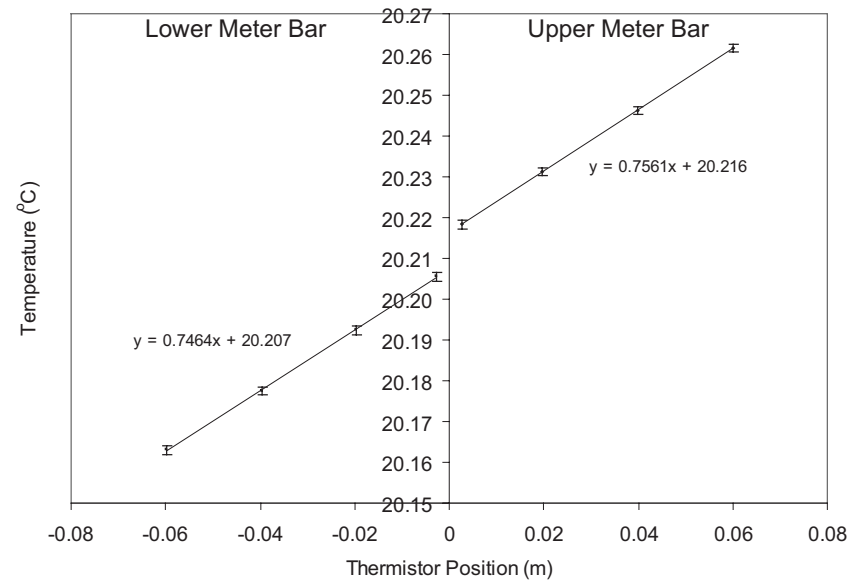

FIG. 5. Low-heat-flux meter-bar temperature distribution during selfcontact, $Q=0.262 \pm 0.006 \mathrm{~W}$, and $P \approx 0.2 \mathrm{MPa}$.

value of $U_{T}$ used for computing that data point. Due to the increase in thermal precision alone, the apparatus developed in the present study allows for an order-of-magnitude improvement in the sensitivity and uncertainty in measured thermal resistance. To emphasize the practical importance of this improvement, the horizontal line plotted in Fig. 4 represents $10 \%$ of the theoretical specific thermal resistance of a TIM having a thickness of $50 \mu \mathrm{m}$ and a thermal conductivity of $5 \mathrm{~W} / \mathrm{m} \mathrm{K}$. Based on the assumptions of this simplified analysis, this demonstrates that, in order to accurately characterize the performance of thin bondline, high-performance TIMs, the instrumentation and techniques presented in the present study are indeed essential.

\section{RESULTS AND DISCUSSION}

In order to validate the sensitivity and precision of the test facility, self-contact interface tests were performed to characterize system performance for low thermal resistance scenarios, where the specific thermal resistance of the TIM would be on the order of magnitude of the self-contact resistance of the meter bars themselves.

The temperature distribution in the meter bars during self-contact is shown in Fig. 5. This extremely low-power case $(Q=0.262 \pm 0.006 \mathrm{~W})$ demonstrates the linearity of the meter bars and temperature sensitivity of the instrumentation. The linear fits to the data indicate that a temperature difference between the contacting surfaces was $0.0089 \pm 0.0012 \mathrm{~K}$, while the heat currents in the meter bars agree to within $0.4 \%$, demonstrating accurate energy balance. As the heater power is increased, these uncertainties drop significantly. Indeed, over a large range of input powers, the calculated heat fluxes between the two meter bars balance within their computed uncertainties, as illustrated in Fig. 6.

The change in specific thermal resistance and electrical contact resistance for the bare, dry meter bars in self-contact is shown as a function of pressure for $Q \approx 4.4 \mathrm{~W}$ in Fig. 7 . Here one can observe a marked decrease in both resistances initially as the contact pressure is increased. The trend in the change in specific thermal resistance with pressure and the overall magnitude correspond well with the data presented

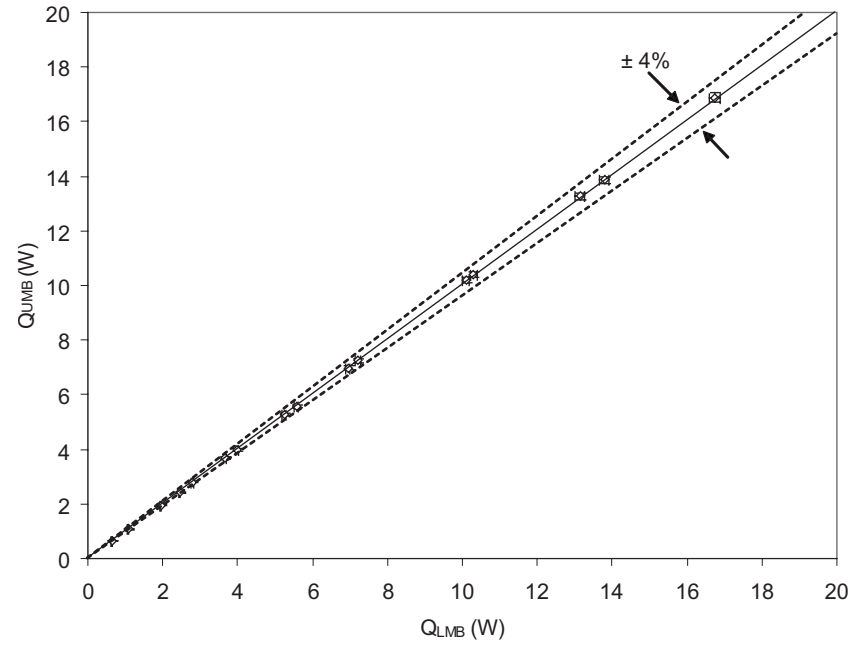

FIG. 6. Energy balance between upper and lower meter bars for a range of powers

by Yovanovich. ${ }^{36} \mathrm{~A}$ minimum dry contact resistance of $2.71 \times 10^{-5} \mathrm{~m}^{2} \mathrm{~K} / \mathrm{W}$ was measured with calculated uncertainty of $1.8 \%$. The trend in electrical contact resistance corresponds well with the results of Misra and Nagaraju, ${ }^{24}$ given the somewhat larger contact area used in the present study. The lowest electrical resistance measured was $320 \mu \Omega$, with an uncertainty of $0.15 \%$. Of this, $5.44 \mu \Omega$ (or $3.4 \%$ of this value) can be attributed to the bulk resistance of the $\mathrm{CuW}$ meter bars.

A single drop of mineral oil was compressed between the meter bars at a pressure of approximately $3 \mathrm{MPa}$ to achieve a lower thermal resistance by displacing any air in the microscopic voids of the contact zone. A comparison between the specific thermal contact resistance for the meter bars in dry contact and using oil as a TIM with heat flux is shown in Fig. 8. Here, the inclusion of oil as a TIM reduces the thermal contact resistance from approximately $2.75 \times 10^{-5}$ to $5 \times 10^{-6} \mathrm{~m}^{2} \mathrm{~K} / \mathrm{W}$. Also demonstrated in Fig. 8 is the reduction in calculated uncertainty with increasing heat flux. The lowest specific thermal resistance measured was of $4.68 \times 10^{-6} \mathrm{~m}^{2} \mathrm{~K} / \mathrm{W}$ with an uncertainty of $2.7 \%$ using a heat transfer rate of $16.8 \mathrm{~W}$. For both the dry contact

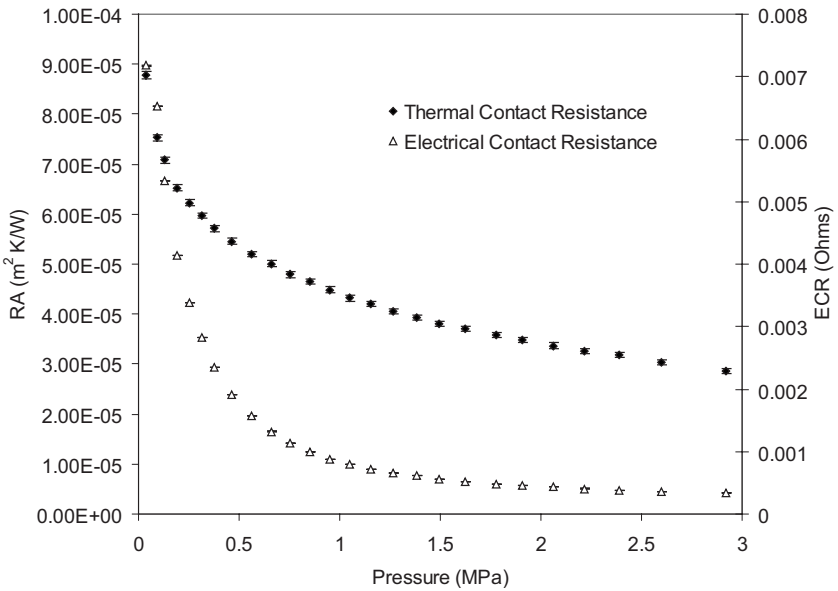

FIG. 7. Change in specific thermal resistance and electrical contact resistance with pressure for dry self-contact, $Q \approx 4.4 \mathrm{~W}$. 


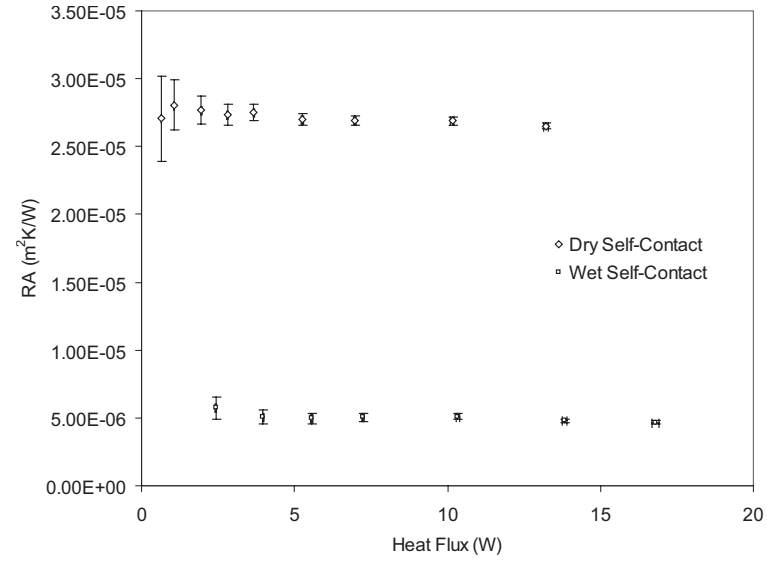

FIG. 8. Specific thermal resistance as a function of heat flux for dry and wet self-contact $(P \approx 3 \mathrm{MPa})$.

and wet contact results, the slight decrease in specific thermal resistance with heat flux can be attributed to a small pressure increase due to system thermal expansion as the overall temperatures increase.

A commercially available graphite TIM nominally 0.125 $\mathrm{mm}$ thick was characterized using this apparatus. Specific thermal resistance and electrical resistance as a function of pressure for this TIM are shown in Fig. 9. For comparison, the specific thermal resistance and electrical resistances from Fig. 8 of the meter bars in dry self-contact are also shown in this figure. These results indicate that the specific thermal resistance of the TIM is of the same order of magnitude as the dry contact resistance of the meter bars themselves. This serves to highlight the aforementioned inherent difficulty of discerning the thermal contact resistance between the meter bars and the TIM and the bulk thermal resistance of the TIM itself. Electrically, however, the bulk resistance of the TIM and meter bars can be considered negligible compared to the electrical contact resistances. The bulk electrical resistance for this $0.125 \mathrm{~mm}$ graphite pad was calculated as approximately $1 \mu \Omega$, and, since the electrical resistance measurement with the TIM is considerably higher than that of the dry self-contact, one can infer that the thermal contact resistance between the TIM and the meter bars plays the more important role in the overall measured specific thermal resistance.

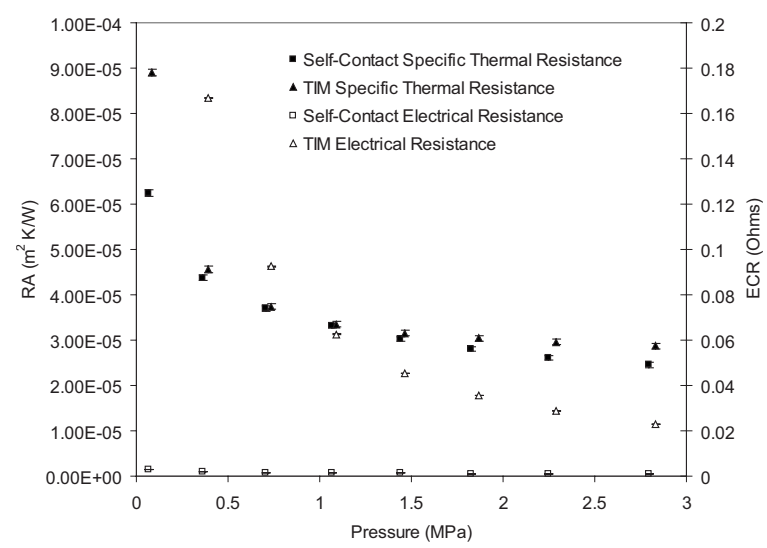

FIG. 9. Change in specific thermal resistance and electrical contact resistance with applied pressure for a graphite.

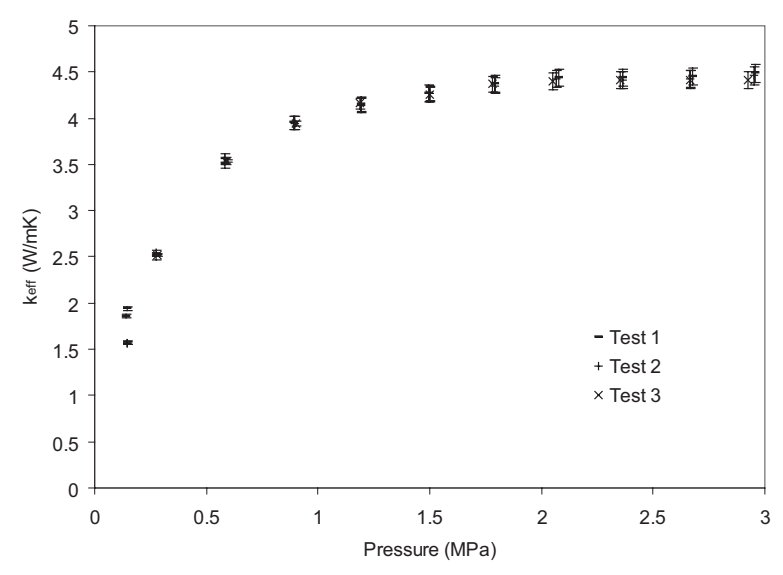

FIG. 10. Change in effective thermal conductivity with applied pressure for a graphite TIM during three tests.

This ability to simultaneously measure thermal and electrical resistance was developed in order to characterize the thermal contact resistance of a MMT-TIM. ${ }^{7}$ A correlation relating the electrical and thermal contact resistances as a function of pressure has been developed for silver MMT-TIMs, thereby allowing a straightforward electrical conductivity measurement to be used to indirectly distinguish the thermal contact resistance from the bulk thermal resistance of the MMT-TIM or any other electrically conductive material. ${ }^{37}$

The change in apparent thermal conductivity of the graphite TIM as a function of pressure is shown in Fig. 10. As the contact resistance between the meter bars and the TIM diminished, the apparent thermal conductivity was seen to asymptotically approach the bulk thermal conductivity value of the TIM, which was approximately $4.5 \mathrm{~W} / \mathrm{m} \mathrm{K}$. Results from two subsequent tests are also shown in Fig. 10 and demonstrate the repeatability of this measurement. At the highest pressures, the measured effective thermal conductivity of the graphite pad exhibited repeatability of approximately $1 \%$.

\section{CONCLUSIONS}

An apparatus with unprecedented precision and sensitivity has been built for the performance characterization of TIMs. This apparatus allows for the precise measurement of specific thermal resistance and apparent thermal conductivity of a TIM under a range of pressures and temperatures, simultaneous with electrical resistance measurements.

Thermal and electrical contact resistance measurements of the meter bars in dry and wet self-contact demonstrated the sensitivity and precision for low-level measurements. The lowest specific thermal resistance measured was 4.68 $\times 10^{-6} \mathrm{~m}^{2} \mathrm{~K} / \mathrm{W}$ with an uncertainty of $2.7 \%$ at an input power of $16.8 \mathrm{~W}$. It is difficult to make direct, qualitative comparisons against the precisions and uncertainties reported in previous studies due to the paucity of uncertainty analysis presented. However, the thermal precision of the current setup alone indicates an order-of-magnitude improvement in precision and sensitivity over any previous investigation. This sensitivity will enable the development and characterization of new, optimized TIMs with thermal impedance values sufficiently low to meet the demanding requirements of 
the International Technology Roadmap for Semiconductors roadmap. ${ }^{38}$ Furthermore, simultaneous measurements of electrical contact resistance will allow for the indirect measurement of the thermal contact resistance of next-generation MMT-TIMs.

The specific thermal resistance, electrical contact resistance, and effective thermal conductivity of a commercially available graphite pad were measured over a range of pressures and demonstrated a correlation between thermal and electrical contact resistance. Due to the difficulty in discerning the difference between the TIM thermal contact resistance with the meter bars and its bulk thermal resistance, it is suggested that further testing of electrically conductive TIMs could yield information about the nature of thermal contact resistance variations with respect to electrical resistance during compression and deformation.

\section{ACKNOWLEDGMENTS}

The authors wish to gratefully acknowledge Dr. Peter Teertstra of the University of Waterloo, Canada for his helpful advice during the planning stages and Rich LaGrotta of Acatel-Lucent for funding the development of this apparatus.

\section{NOMENCLATURE}

$$
\begin{aligned}
A & =\text { Area, } \mathrm{m}^{2} \\
k & =\text { Thermal conductivity, } \mathrm{W} / \mathrm{m} \mathrm{K} \\
d & =\text { Thermistor axial position, } \mathrm{m} \\
\mathrm{ECR} & =\text { Electrical contact resistance, } \Omega \\
f & =\text { Fractional uncertainty } \\
L & =\text { Thickness, } \mathrm{m} \\
m & =\text { Temperature gradient, } \mathrm{K} / \mathrm{m} \\
Q & =\text { Heat transfer rate, } \mathrm{W} \\
R & =\text { Thermal resistance, } \mathrm{K} / \mathrm{W} \\
R A & =\text { Specific thermal resistance or thermal imped- } \\
& \text { ance, } \mathrm{m}^{2} \mathrm{~K} / \mathrm{W} \\
T & =\text { Temperature, } \mathrm{K} \\
\Delta T & =\text { Extrapolated temperature difference, } \mathrm{K} \\
U & =\text { Uncertainty } \\
c & =\text { Measured quantity } \\
\Delta x & =\text { Temperature sensor spacing, } \mathrm{m} \\
Z & =\text { Calculated quantity }
\end{aligned}
$$

Subscripts

$$
\begin{aligned}
a & =\text { Upper meter-bar surface } \\
b & =\text { Lower meter-bar surface } \\
d & =\text { Thermistor position } \\
\text { eff } & =\text { Effective } \\
\mathrm{ext} & =\text { Extrapolation } \\
\mathrm{mb} & =\text { Meter bar } \\
\mathrm{lmb} & =\text { Lower meter bar } \\
\mathrm{umb} & =\text { Upper meter bar } \\
n & =\text { Thermistor noise } \\
\mathrm{cal} & =\text { Calibration temperature }
\end{aligned}
$$

${ }^{1}$ B. Smith, T. Brunschwiler, and B. Michel, Microelectron. J. 40(9), 13791386 (2008).

${ }^{2}$ R. Linderman, T. Brunschwiler, B. Smith, and B. Michel, Proc. THERMINIC (THERMINIC, Budapest, 2007), pp. 129-134.

${ }^{3}$ A. Ziaei and S. Demoustier, Proc. THERMINIC (THERMINIC, Rome, 2008), pp. 153-155.

${ }^{4}$ J. Liu, B. Michel, M. Rencz, C. Tantolin, C. Sarno, R. Miessner, K. V.
Schuett, X. Tang, S. Demoustier, and A. Ziaei, Proc. THERMINIC (THERMINIC, Rome, 2008), pp. 156-161.

${ }^{5}$ J. J. W. Tzeeng, T. W. Weber, and D. W. Krassowski, Proc. 16th IEEE SEMI-THERM Symposium (SEMI-THERM, San Jose, 2000), pp. 174181.

${ }^{6}$ P. Teertstra, Proc. InterPACK 2007 (InterPACK, Vancouver, 2007), pp. 381-388.

${ }^{7}$ R. Kempers, R. Frizzell, A. Lyons, and A. J. Robinson, Proc. InterPACK 2009 (InterPACK, San Francisco, 2009 ), Paper No. IPACK2009-89366.

${ }^{8}$ E. G. T. Bosch and C. J. M. Lasance, Proc. 16th IEEE SEMI-THERM Symposium (SEMI-THERM, San Jose, 2000), pp. 167-173.

${ }^{9}$ C. J. M. Lasance and C. Lacaze, Proc. 12th IEEE SEMI-THERM Symposium (SEMI-THERM, Phoenix, 1996), pp. 43-45.

${ }^{10}$ C. J. M. Lasance, C. T. Murray, D. L. Saums, and M. Rencz, Proc. 22nd IEEE SEMI-THERM Symposium (SEMI-THERM, Dallas, 2006), pp. 4249.

${ }^{11}$ M. R. Rencz and V. Székely, IEEE Trans. Compon. Packag. Technol. 25, 547 (2002).

${ }^{12}$ M. Rencz, V. Székely, G. Farkas, and B. Courtois, Proc. IEEE InterSociety Conference on Thermal Phenomena (ITHERM, San Diego, 2002), pp. 136-141.

${ }^{13}$ B. Smith, T. Brunschwiler, and B. Michel, Proc. 13th Workshop on Thermal Issues in ICs and Systems (THERMINIC, Budapest, 2007), pp. 134139.

${ }^{14}$ W. Parker, R. Jenkins, C. Butler, and G. Abbott, J. Appl. Phys. 32, 1679 (1961).

${ }^{15}$ L. Kehoe, P. V. Kelly, and G. M. Crean, Microsyst. Technol. 5, 18 (1998).

${ }^{16}$ P. S. Gaal, M. A. Thermitus, and D. E. Stroe, J. Therm. Anal. Calorim. 78, 185 (2004).

${ }^{17}$ V. Khuu, M. Osterman, A. Bar-Cohen, and M. Pecht, Proc. InterPACK, (InterPACK, Vancouver, 2007), pp. 405-414.

${ }^{18}$ J. P. Gwinn, M. Saini, and R. L. Webb, Proc. IEEE Inter-Society Conference on Thermal Phenomena (ITHERM, San Diego, 2002), pp. 644-650.

19 "Standard test method for thermal transmission properties of thermally conductive electrical insulation materials," ASTM Standard D5470-06, Copyright ASTM International, Conshohocken, PA, 2007.

${ }^{20}$ D. Kearns, Proc. 19th IEEE Semi-Therm Symposium (SEMI-THERM, San Jose, 2003), pp. 129-133.

${ }^{21}$ J. R. Culham, P. Teertstra, I. Savija, and M. M. Yovanovich, Proc. IEEE Inter-Society Conference on Thermal Phenomena (ITHERM, San Diego, 2002), pp. 128-134.

${ }^{22}$ I. Savija, J. R. Culham, and M. M. Yovanovich, Proc. InterPACK (InterPACK, Maui, 2003), pp. 567-574.

${ }^{23}$ V. V. Rao, K. Bapurao, J. Nagaraju, and M. V. Krishna Murthy, Meas. Sci. Technol. 15, 275 (2004).

${ }^{24}$ P. Misra and J. Nagaraju, Rev. Sci. Instrum. 75, 2625 (2004).

${ }^{25}$ V. Singhal, P. J. Litke, A. F. Black, and S. V. Garimella, Int. J. Heat Mass Transfer 48, 5446 (2005).

${ }^{26}$ C. V. Madhusudana, Int. Commun. Heat Mass Transfer 27, 877 (2000).

${ }^{27}$ R. Kempers, P. Kolodner, A. Lyons, and A. J. Robinson, Proc. IEEE ITherm (ITHERM, Orlando, 2008), pp. 221-226.

${ }^{28}$ P. Kolodner, M. Hodes, I. Ewes, and P. Holmes, Proc. InterPACK (InterPACK, San Francisco, 2005), pp. 91-98.

${ }^{29}$ M. A. Kedzierski and J. L. Worthington, Exp. Heat Transfer 6, 329 (1993).

${ }^{30}$ Keithley, Low Level Measurements Handbook: Precision DC Current, Voltage, and Resistance Measurements, 6th ed. (Keithley Instruments, Cleveland, OH, 2004).

${ }^{31}$ A. Wald, Ann. Math. Stat. 11, 284 (1940).

${ }^{32}$ M. S. Bartlett, Biometrics 5, 207 (1949).

${ }^{33}$ W. H. Press, S. A. Teukosky, W. T. Vetterling, and B. P. Flannery, Numerical Recipes in FORTRAN-The Art of Scientific Computing, 2nd ed. (Cambridge University Press, Cambridge, 1992), Chap. 15.

${ }^{34}$ H. H. Ku, NBS Report No. 8677, 1965.

${ }^{35}$ S. J. Kline and F. A. McClintock, Mech. Eng. (Am. Soc. Mech. Eng.) 75, 3 (1953).

${ }^{36}$ M. M. Yovanovich, IEEE Trans. Compon. Packag. Technol. 28, 182 (2005).

${ }^{37}$ R. Kempers, A. Lyons, and A. J. Robinson, Proc. DSL, Rome, Italy, 24-26 June 2009, article DSL 514 (unpublished).

38 "The International Technology Roadmap for Semiconductors 2007 edition: Assembly and packaging," International Technology Roadmap for Semiconductors, 2007. 